Proceedings of the

International Geometry Center

Vol. 13 , no. 3 (2020) pp. 18-32

\title{
Квазі-геодезичні відображення спеціальних псевдоріманових просторів
}

\author{
Курбатова I.М., Піструіл М.I.
}

\begin{abstract}
The article is devoted to the study of a special type of diffeomorphisms of pseudo-Riemannian spaces with an affinor structure.

In [12] we studied mappings of pseudo-Riemannian spaces, which are quasi-geodesic, [16], and at the same time almost geodesic of the second type [17]. By definition, for a quasi-geodesic mapping corresponding to the affinor $F_{i}^{h}$, each geodesic curve in the space $\left(V_{n}, g_{i j}\right)$ is mapped onto the socalled quasi-geodesic curve in another space $\left(\bar{V}_{n}, \bar{g}_{i j}, F_{i}^{h}\right)$. In $[12,18]$ it was assumed that the quasi-geodesic mapping $V_{n}$ onto $\bar{V}_{n}$ satisfies the condition of reciprocity, i.e. the inverse mapping is also quasi-geodesic, corresponding to the same affinor $F_{i}^{h}$. In this case, the affinor satisfies the purely algebraic conditions (consistency with the metric tensors $V_{n}$ and $\bar{V}_{n}$ ). With an almost geodesic mapping of the second type, by definition, each geodesic curve in $\left(V_{n}, g_{i j}, F_{i}^{h}\right)$ is mapped onto an almost geodesic curve in $\left(\bar{V}_{n}, \bar{g}_{i j}\right)$, if the affinor $F_{i}^{h}$ in $V_{n}$ satisfies a certain differential equation. In [12] it is proved that the set of the specified algebraic and differential conditions leads to the fact that the affinor $F_{i}^{h}$ necessarily determines the $e$-structure in $V_{n}$, and elliptic and hyperbolic cases are considered. We call an affinor with such conditions a generalized-recurrent structure (and $V_{n}$ with such a structure, respectively, a generalized-recurrent space). In what follows, we study quasigeodesic mappings of parabolic type generalized-recurrent spaces.

In this paper, we obtain the properties of the Riemannian tensor of generalized-recurrence space associated with the generalized-recurrence vector. It is proved that the class of pseudo-Riemannian spaces with generalized-recurrent structure of parabolic type is closed with respect to the considered mappings, but the vectors of generalized recurrence of spaces $V_{n}$ and $\bar{V}_{n}$ may be distinct. If the generalized recurrence vector is gradient, there is a $K$-structure in the generalized-recurrence space. It is proved that if $K$-space admits a quasigeodesic mapping, which preserves an integrable parabolic type $K$-structure, then this $K$-structure is Kähler (note that an integrable $K$-structure of parabolic type may not be Kähler). The structure of the Riemannian tensor
\end{abstract}

Ключові слова: affinor structure, quasi-geodesic maps

DOI: http://dx.doi.org/10.15673/tmgc.v13i3.1770 
of parabolic type generalized-recurrent space, which admits quasi-geodesic mapping onto a flat space, is found. The components of the metric tensor of such a space in a special coordinate system are given.

Анотація. Стаття присвячена досліженню спеціального типу дифеоморфізмів псевдоріманових просторів з афінорною структурою.

В [12] вивчалися дифеоморфізми псевдоріманових просторів, які $е$ квазі-геодезичними відображеннями [16] і водночас майже геодезичними другого типу [17]. За означенням при квазі-геодезичному відображенні, що відповідає афінору $F_{i}^{h}$, геодезичні лінії простору $\left(V_{n}, g_{i j}\right)$ переходять в так звані квазі-геодезичні лінії іншого простору $\left(\bar{V}_{n}, \bar{g}_{i j}, F_{i}^{h}\right)$. В $[12,18]$ вважалося, що КГВ $V_{n}$ на $\bar{V}_{n}$ задовольняє умові взаємності, тобто зворотне відображення також $є$ квазі-геодезичним, відповідаючим тому ж афінору $F_{i}^{h}$. При цьому умови на афінор носять суто алгебраїчний характер (узгодженість з метричними тензорами $V_{n}$ i $\bar{V}_{n}$ ). При майже геодезичному відображенні другого типу за означенням геодезичні лінії $\left(V_{n}, g_{i j}, F_{i}^{h}\right)$ переходять в майже геодезичні лінії $\left(\bar{V}_{n}, \bar{g}_{i j}\right)$, якщо афінор $F_{i}^{h}$ в $V_{n}$ задовольняє певним диференціальним рівнянням. В [12] доведено, що сукупність вказаних алгебраїчних і диференціальних умов приводить до того, що афінор $F_{i}^{h}$ необхідно визначає на $V_{n} e$-структуpy, i розглянуто еліптичний та гіперболічний випадки. Ми називаємо афінорну структуру з такими умовами узагальнено-рекурентною (а $V_{n}$ з такою структурою, відповідно, узагальнено-рекурентним простором) i обираємо для дослідження квазі-геодезичні відображення узагальненорекурентних просторів параболічного типу.

В даній статті знайдено зв'язок тензора Рімана узагальнено-рекурентного простору з вектором узагальненої рекурентності. Доведено, що клас псевдо-ріманових просторів з узагальнено-рекурентною структурою параболічного типу замкнений відносно розглядуваних відображень, але при цьому вектори узагальненої рекурентності просторів $V_{n}$ i $\bar{V}_{n}$ можуть виявитись не тотожними. Якщо вектор узагальненої рекурентності градієнтний, в узагальнено-рекурентному просторі існує $K$-структура. Доведено, що якщо $K$-простір допускає квазі-геодезичне відображення зі збереженням інтегровної $K$-структури параболічного типу, то ця $K$ структура $є$ келеровою, хоча сама по собі інтегровна $K$-структура параболічного типу може не бути келеровою. Знайдена структура тензора Рімана узагальнено-рекурентного простору параболічного типу, який допускає квазі-геодезичне відображення на плоский простір. Наведено компоненти метричного тензора такого простору в спеціальній системі координат.

\section{1. ВСтУП}

Одним з найбільш змістовних (в прикладному сенсі) узагальненням поняття геодезичного відображення [17] ріманових просторів є запропоноване академіком А. З. Петровим квазі-геодезичне відображення (надалі КГВ) псевдоріманових просторів $V_{4}$ сігнатури Мінковського [16]. 
При цьому рух вільної частинки в одному фізичному полі моделюється рухом частинки в іншому полі під дією деякої сили (наприклад, на рухомий електричний заряд в електромагнітному полі діє сила Лоренца). 3 геометричної точки зору це означає, що геодезичні лінії простору $\left(V_{4}, g_{i j}\right)$ переходять в так звані квазі-геодезичні лінії іншого простору $\left(\bar{V}_{4}, \bar{g}_{i j}\right)$.

А. З. Петров знайшов основні рівняння квазі-геодезичного відображення $V_{4}$ на $\bar{V}_{4}$ в спільній за відображенням системі координат у вигляді:

$$
\begin{aligned}
& \bar{\Gamma}_{i j}^{h}(x)=\Gamma_{i j}^{h}(x)+\psi_{(i}(x) \delta_{j)}^{h}+\phi_{(i}(x) F_{j)}^{h}(x), \\
& \bar{g}_{i \alpha} F_{j}^{\alpha}=-\bar{g}_{j \alpha} F_{i}^{\alpha}, \quad i, h, j, \ldots=1,2,3,4,
\end{aligned}
$$

де $\Gamma_{i j}^{h}, \bar{\Gamma}_{i j}^{h}$ - компоненти об'єктів зв'язності $V_{4}, \bar{V}_{4}$, відповідно, $\psi_{i}(x), \phi_{i}(x)$ - деякі ковектори, $F_{i}^{h}$ - афінор, а дужками позначена операція симетрування по відповідних індексах.

Дослідженню КГВ при деяких додаткових умовах присвячені роботи $[13,14,12]$. Там поняття КГВ узагальнено на випадок псевдоріманових просторів довільної сігнатури і розмірності, причому в [18] вважається, що КГВ $V_{n}$ на $\bar{V}_{n}$ задовольняє умові взаємності, тобто зворотне відображення також є квазі-геодезичним, відповідаючим тому ж афінору $F_{i}^{h}$. Отже в $V_{n}$ також виконуються співвідношення

$$
g_{i \alpha} F_{j}^{\alpha}=-g_{j \alpha} F_{i}^{\alpha},
$$

Іншим найбільш природним з геометричної точки зору узагальненням геодезичного відображення є майже геодезичні відображення афіннозв'язних і ріманових просторів [17], введені до розгляду Н. С. Сінюковим. В останні десятиліття з'явилося багато наукових робіт $[2,1,7,10]$, що присвячені дослідженню майже геодезичних відображень і містять цікаві результати.

Класи квазі-геодезичних і майже геодезичних відображень мають істотний перетин, якому належать, наприклад голоморфно-проективні відображення келерових просторів [8, 3, 5, 6, 9, 4]. Найбільш близькими до КГВ є майже геодезичні відображення 2-го типу [17] з основними рівняннями (1.1) (при $i, h, j, \ldots=1,2, \ldots, n)$, в яких афінор задовольняє диференціальним умовам

$$
F_{(i, j)}^{h}+F_{\alpha}^{h} F_{(i}^{\alpha} \phi_{j)}=p_{(i} \delta_{j)}^{h}+q_{(i} F_{j)}^{h},
$$

де $p_{i}, q_{i}$ - деякі ковектори, а "," - знак коваріантної похідної відносно зв'язності простору $V_{n}$.

Ми розглядаємо відображення псевдоріманових просторів, що належать зазначеному перетину, а саме $\mathrm{K} \Gamma \mathrm{B}$, при якому афінор $F_{i}^{h}$ задовольняє диференціальним умовам (1.3). 
В [12] доведено, що афінор $F_{i}^{h}$, який задовольняє (1.3) і узгоджений з метрикою $g_{i j}$ у вигляді (1.2), необхідно визначає на $V_{n}$ так звану $e$ структуру [17], тобто мають місце співвідношення

$$
F_{\alpha}^{h} F_{i}^{\alpha}=e \delta_{i}^{h}
$$

де $e=-1,+1$ або 0 . При цьому $e$-структуру називають

- еліптичною, якщо $e=-1$,

- гіперболічною, коли $e=+1$,

- $m$-параболічною при $e=0, \operatorname{rank} F=m,(2 m<n)$ та

- параболічною при $e=0, \operatorname{rank} F=m,(2 m=n)$.

В $[12,18]$ вивчалися КГВ псевдоріманових просторів $\left(V_{n}, g_{i j}, F_{i}^{h}\right)$, на яких афінор задає $K$-структуру [11] еліптичного або гіперболічного типів, тобто задовольняє (1.4) при $e=+1,-1$, а також диференціальним умовам

$$
F_{(i, j)}^{h}=0 .
$$

Метою нашого дослідження будуть квазі-геодезичні відображення псевдоріманових просторів довільної розмірності, які задовольняють умові взаємності і водночас є майже геодезичними відображеннями 2го типу.

Дослідження ведеться в тензорній формі, локально, в класі дійсних досить гладких функцій.

\section{2. ПОНЯТТЯ І ВЛАСТИВОСТІ УЗАГАЛЬНЕНО-РЕКУРЕНТНОЇ СТРУКТУРИ}

$1^{\circ}$. Розглянемо простір $\left(V_{n}, g_{i j}, F_{i}^{h}\right)$, в якому афінор $F_{i}^{h}$ задовольняе умовам (1.3) і (1.4):

$$
\begin{aligned}
F_{(i, j)}^{h}+F_{\alpha}^{h} F_{(i}^{\alpha} \phi_{j)} & =p_{(i} \delta_{j)}^{h}+q_{(i} F_{j)}^{h}, \\
F_{\alpha}^{h} F_{i}^{\alpha} & =e \delta_{i}^{h} .
\end{aligned}
$$

Будемо називати таку афінорну структуру узагальнено-рекурентною (еліптичного, гіперболічного або параболічного типу залежно від значення $е$ ), а сам простір $V_{n}$ - узагальнено-рекурентним відповідного типу. Зауважимо, що $K$-структура є окремим випадком узагальненорекурентної.

Іншим окремим випадком узагальнено-рекурентної структури є введена в [15] рекурентно-параболічна афінорна структура, яка визначається умовами

$$
F_{\alpha}^{h} F_{i}^{\alpha}=0, \quad g_{i \alpha} F_{j}^{\alpha}=-g_{j \alpha} F_{i}^{\alpha}, \quad F_{i, j}^{h}=q_{j} F_{i}^{h} .
$$

Розглянемо властивості узагальнено-рекурентної структури параболічного типу.

$2^{\circ}$. $\quad$ По-перше доведемо 
Твердження 2.1. Якщо абінорна структура $F_{i}^{h}$ узагалънено-рекурентного простору параболічного типу $\left(V_{n}, g_{i j}, F_{j}^{h}\right)$ узгоджена з метричним тензором $g_{i j}$ у бормі (1.2):

$$
g_{i \alpha} F_{j}^{\alpha}=-g_{j \alpha} F_{i}^{\alpha} \text {, }
$$

то диференціальні рівняння (1.3) набувають вигляду

$$
F_{(i, j)}^{h}=F_{(i}^{h} q_{j)}
$$

Доведення. Дійсно, опускаючи в (1.3) індекс $h$ за допомогою метричного тензора $g_{i j}$ з урахуванням

$$
F_{\alpha}^{h} F_{i}^{\alpha}=0
$$

отримаємо:

$$
F_{h(i, j)}=g_{h(j} p_{i)}+F_{h(j} q_{i)}
$$

Проциклюємо цю рівність по $h, i, j$ з урахуванням (1.2) і згорнемо з $g^{j h}$ по індексах $j, h$. В результаті маємо

$$
p_{i}=0 \text {. }
$$

Отже за умов (1.2) і (2.1) рівність (1.3) набуває вигляду

$$
F_{(i, j)}^{h}=F_{(i}^{h} q_{j)}
$$

Твердження доведено.

Умовимось називати вектор $q_{i}$ в (2.2) вектором узагальненоӥ рекурентності структури $F_{i}^{h}$.

Зауваження 2.2. При $q_{i}=0$ узагальнено-рекурентна структура $є K$ структурою, а за умови градієнтності $q_{i}$ узагальнено-рекурентний прос$\operatorname{sip}\left(V_{n}, g_{i j}, F_{i}^{h}\right)$ допускає $K$-структуру $\tilde{F}_{i}^{h}=e^{-q} F_{i}^{h}$.

$3^{\circ}$. Домовимося надалі операцію згортання з афінором називати сполученням за відповідним індексом і позначати наступним чином:

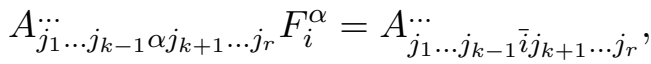

$$
\begin{aligned}
& A_{\ldots}^{j_{1} \ldots j_{k-1} \alpha j_{k+1} \ldots j_{r}} F_{\alpha}^{h}=A_{\ldots}^{j_{1} \ldots j_{k-1}} \bar{h} j_{k+1} \ldots j_{r} .
\end{aligned}
$$

$4^{\circ}$. Інтегровна параболічна e-cmpуктура $F_{i}^{h}$ в деякому околі кожної точки $V_{n}$ за рахунок вибору системи координат (адаптованої до афінора), може бути приведена до вигляду

$$
\left(F_{i}^{h}\right)=\left(\begin{array}{cc}
0 & 0 \\
I_{m} & 0
\end{array}\right),
$$

де $I_{m}$ - одинична матриця порядку $m=\frac{n}{2}$. 
Надалі нам буде корисний допоміжний тензор $A_{i}^{h}$, який в адаптованій системі координат визначається матрицею

$$
\left(A_{i}^{h}\right)=\left(\begin{array}{cc}
P & I_{m} \\
-P^{2} & -P
\end{array}\right),
$$

де $P$ - довільна квадратна матриця порядку $m$.

При цьому, очевидно, за необхідністю

$$
F_{\alpha}^{\beta} A_{\beta}^{\alpha}=m, \quad A_{\alpha}^{h} A_{i}^{\alpha}=0, \quad F_{\alpha}^{h} A_{i}^{\alpha}+A_{\alpha}^{h} F_{i}^{\alpha}=\delta_{i}^{h} .
$$

$5^{\circ}$. Доведемо

Твердження 2.3. Тензор Рімана узагальнено-рекурентного простору параболічного типу $\left(V_{n}, g_{i j}, F_{i}^{h}\right)$ задовольняе співвідношенням

$$
3\left(R_{\bar{h} j k i}+R_{h \bar{j} k i}+R_{h j \bar{k} i}+R_{h j k \bar{i}}\right)=2 Q_{j h k i}+Q_{j k h i}-Q_{h k j i},
$$

$\partial e$

$$
Q_{h j k i}=q_{[h, j]} F_{k i}+q_{[k, i]} F_{h j} .
$$

Доведення. Опустимо в (2.2) індекс $h$ в $V_{n}$ і продиференціюємо коваріантно по $x^{k}$ отримані співвідношення:

$$
F_{h i, j k}+F_{h j, i k}=\left(F_{h(i} q_{j)}\right)_{, k} .
$$

3 огляду на (2.2) цей вираз може бути переписано інакше:

$$
F_{h i,[j k]}+F_{h j,[i k]}-F_{h k,(i j)}=\left(F_{h(i} q_{j)}\right)_{, k}-\left(F_{h(i} q_{k)}\right)_{, j}-\left(F_{h(k} q_{j)}\right)_{, i}
$$

або

$$
\begin{aligned}
F_{h i,[j k]}+ & F_{h j,[i k]}-F_{h k,(i j)}= \\
& F_{h i, k} q_{j}+F_{h j, k} q_{i}+F_{h i} q_{j, k}+F_{h j} q_{i, k}-F_{h i, j} q_{k}-F_{h i} q_{k, j}- \\
& \quad-F_{h k} q_{i, j}-F_{h k, j} q_{i}-F_{h k, i} q_{j}-F_{h k} q_{j, i}-F_{h j, i} q_{k}-F_{h j} q_{k, i} .
\end{aligned}
$$

Симетруючи тут по $h, k, з$ урахуванням тотожності Річчі і (2.2) та після зведення подібних маємо:

$$
\begin{aligned}
R_{\bar{i} h j k}-R_{\overline{h i j k}}+R_{\bar{j} h i k} & -R_{\bar{h} j i k}+ \\
& +R_{\bar{i} k j h}-R_{\bar{k} i j h}+R_{\bar{j} k i h}-R_{\bar{k} j i h}=Q_{j k h i}+Q_{j h k i},
\end{aligned}
$$

де позначено

$$
Q_{j h k i}=q_{[j, h]} F_{k i}+q_{[k, i]} F_{j h} .
$$

Альтернуючи результат по $h, j$ і застосовуючи тотожність Біанкі, нарешті отримуємо

$$
3\left(R_{\bar{h} j k i}+R_{h \bar{j} k i}+R_{h j \bar{k} i}+R_{h j k \bar{i}}\right)=2 Q_{j h k i}+Q_{j k h i}-Q_{h k j i} .
$$

Твердження доведено. 
При $q_{i}=0$ узагальнено-рекурентна структура $F_{i}^{h} \in K$-структурою, отже в $K$-просторі тензор Рімана характеризується властивістю

$$
R_{\bar{h} j k i}+R_{h \bar{j} k i}+R_{h j \bar{k} i}+R_{h j k \bar{i}}=0,
$$

або після піднімання індекса $h$ в $V_{n}$

$$
-R_{j k i}^{\bar{h}}+R_{\overline{j k i}}^{h}+R_{j \overline{k i}}^{h}+R_{j k \bar{i}}^{h}=0
$$

Має місце

Теорема 2.4. В узагалънено-рекурентному просторі параболічного тиny $\left(V_{n}, g_{i j}, F_{j}^{h}\right)$ рівність

$$
R_{\bar{h} j k i}+R_{h \bar{j} k i}+R_{h j \overline{k i}}+R_{h j k \bar{i}}=0
$$

виконуетъся тоді і тільки тоді, коли вектор узагальненої рекурентності $q_{i}$ градієнтний, тобто в $V_{n}$ існуе $K$-структура $\tilde{F}_{i}^{h}=e^{-q} F_{i}^{h}$.

Доведення. Дійсно, якщо вектор узагальненої рекурентності $q_{i}$ градієнтний, то $Q_{h j k i}=0$ і (2.5) набуває вигляду

$$
R_{\bar{h} j k i}+R_{h \bar{j} k i}+R_{h j \bar{k} i}+R_{h j k \bar{i}}=0,
$$

звідки, до речі, після згортання з $g^{j k}$ по індексах $j, k$ випливає, що

$$
R_{\bar{h} i}=-R_{h \bar{i}}
$$

де $R_{h i}$ - тензор Річчі.

3 іншого боку, можно довести, що якщо тензор Рімана узагальненорекурентного простору параболічного типу задовольняє (2.7), то вектор узагальненої рекурентності $q_{i}$ необхідно градієнтний.

Дійсно, з (2.5) за умови (2.7) маємо:

$$
2 Q_{j h k i}+Q_{j k h i}-Q_{h k j i}=0 .
$$

Із означення тензора $Q_{j h k i}$ бачимо, що $Q_{j h k i}=-Q_{h j k i}$, тому симетрування останнього співвідношення по індексах $j, k$ дає нам

$$
Q_{j h k i}+Q_{k h j i}=0
$$

або

$$
q_{j h} F_{k i}+q_{k i} F_{j h}+q_{k h} F_{j i}+q_{j i} F_{k h}=0, \quad q_{i j}=q_{[i, j]} .
$$

Введемо до розгляду вектори $a^{i} \mathrm{i} b^{i}$ такі, що $a^{\alpha} F_{\alpha i} \neq 0, a^{\alpha} F_{\alpha \beta} b^{\beta}=1 \mathrm{i}$ згорнемо послідовно (2.8) з $a^{j}$ по $j, з a^{h} b^{i}$ по $h, i, з b^{k}$ по $k$ :

$$
\begin{gathered}
a^{\alpha} q_{\alpha h} F_{k i}+q_{k i} a^{\alpha} F_{\alpha h}+q_{k h} a^{\alpha} F_{\alpha i}+a^{\alpha} q_{\alpha i} F_{k h}=0 \\
a^{\alpha} q_{\alpha k}+a^{\alpha} b^{\beta} q_{\alpha \beta} a^{\gamma} F_{\gamma k}=0 \\
2 a^{\alpha} b^{\beta} q_{\alpha \beta}=0 .
\end{gathered}
$$


3 огляду на дві останні рівності (2.9) приймає вигляд

$$
q_{k i} a^{\alpha} F_{\alpha h}+q_{k h} a^{\alpha} F_{\alpha i}=0 .
$$

Тепер згорнемо (2.10) послідовно з $b^{h}$ по індексу $h$ та з $b^{i}$ по $i$ :

$$
\begin{gathered}
q_{k i}+b^{\beta} q_{k \beta} a^{\alpha} F_{\alpha i}=0, \\
2 b^{\beta} q_{k \beta}=0 .
\end{gathered}
$$

Звідси маємо

$$
q_{k i}=q_{[k, i]}=0
$$

Отже при

$$
R_{\bar{h} j k i}+R_{h \bar{j} k i}+R_{h j \bar{k} i}+R_{h j k \bar{i}}=0
$$

вектор $q_{i}$ - градієнтний, тобто в узагальнено-рекурентному просторі $V_{n}$ існує $K$-структура $\tilde{F}_{i}^{h}=e^{-q} F_{i}^{h}$.

$6^{\circ}$. Розглянемо випадок, коли узагальнено-рекурентна структура параболічного типу інтегровна. Доведемо

Твердження 2.5. Абінорна структура узагальнено-рекурентного простору параболічного типу $\left(V_{n}, g_{i j}, F_{j}^{h}\right)$ за умови їі інтегровності характеризуетъся властивостями

$$
\begin{gathered}
F_{i, \alpha}^{\alpha}=0, \\
F_{\bar{j}, i}^{h}=F_{j, \bar{i}}^{h}=F_{j, i}^{\bar{h}}=0 .
\end{gathered}
$$

Доведення. Дійсно, як відомо, один з критеріїв інтегровності e-структури стверджує, що в цьому разі дорівнює нулю їі тензор Нейєнхейса [17]:

$$
N_{i j}^{h}=F_{\bar{i}, j}^{h}-F_{j, \bar{i}}^{h}-F_{\bar{j}, i}^{h}+F_{i, \bar{j}}^{h}=0 .
$$

Оскільки з огляду на (2.1) та (2.2)

$$
F_{\bar{i}, j}^{h}=-F_{i, j}^{\bar{h}}=F_{j, i}^{\bar{h}}=-F_{\bar{j}, i}^{h},
$$

то (2.11) набувають вигляду

$$
4 F_{\bar{j}, i}^{h}-q_{\bar{j}} F_{i}^{h}+q_{\bar{i}} F_{j}^{h}=0
$$

або, що те ж саме,

$$
4 F_{h \bar{j}, i}-q_{\bar{j}} F_{h i}+q_{\bar{i}} F_{h j}=0 .
$$

Після симетрування по $h, i$ звідси випливає

$$
q_{\bar{i}} F_{h j}+q_{\bar{h}} F_{i j}=0
$$

або

$$
q_{i} F_{h}^{j}+q_{\bar{h}} F_{i}^{j}=0
$$


Згортаючи отримане рівняння з $q_{j}$ по індексу $j$, знаходимо

$$
q_{\bar{i}} q_{\bar{h}}=0
$$

звідки

$$
q_{\bar{i}}=0 \text {. }
$$

Тоді з (2.2) випливає бездивернійність афінора

$$
F_{i, \alpha}^{\alpha}=0
$$

а $3(2.11)$ та $(2.12)$

Твердження доведено.

$$
F_{\bar{j}, i}^{h}=F_{j, i}^{h}=F_{j, i}^{\bar{h}}=0
$$

Неважко перевірити, що для інтегровності узагальнено-рекурентної структури достатньо умов $F_{j, \bar{i}}^{h}=0, F_{i, \alpha}^{\alpha}=0$ (для $K$-структури лише $\left.F_{j, \bar{i}}^{h}=0\right)$.

Зауважимо, що на відміну від гіперболічного та еліптичного типів, інтегровна узагальнено-рекурентна структура параболічного типу (зокрема параболічна $K$-структура) не є обов'язково келеровою, тобто із співвідношень (2.14) не випливає коваріантна сталість афінора $F_{i}^{h}$.

Домовимось надалі в цій статті роглядати тільки інтегровну афінорну структуру.

\section{3. КГВ УЗАГАЛЬНЕНО-РЕКУРЕНТНИХ ПРОСТОРІВ ПАРАБОЛІЧНОГО типу}

$1^{\circ}$. Нехай узагальнено-рекурентний псевдорімановий простір параболічного типу $\left(V_{n}, g_{i j}, F_{i}^{h}\right)$ допускає нетривіальне КГВ на простір $\left(\bar{V}_{n}, \bar{g}_{i j}\right)$, яке задовольняє умові взвємності. Тоді в спільній за відображенням системі координат $\left(x^{i}\right)$ виконуються основні рівняння

$$
\begin{gathered}
\bar{\Gamma}_{i j}^{h}(x)=\Gamma_{i j}^{h}(x)+\psi_{(i}(x) \delta_{j)}^{h}+\phi_{(i}(x) F_{j)}^{h}(x), \\
F_{i j}=-F_{j i}, \quad F_{i j}=g_{i \alpha} F_{j}^{\alpha}, \quad \bar{F}_{i j}=-\bar{F}_{j i}, \quad \bar{F}_{i j}=\bar{g}_{i \alpha} F_{j}^{\alpha}, \\
F_{\alpha}^{h} F_{i}^{\alpha}=0, \\
F_{(i, j)}^{h}=F_{(i}^{h} q_{j} .
\end{gathered}
$$

Зауважимо, що коли в (3.1) $\phi_{i} \neq 0$ і $\psi_{i}=0$, квазі-геодезичне відображення називають канонічним. В даній статті досліджуються КГВ, які не є канонічними. Має місце

Теорема 3.1. Образом узагальнено-рекурентного простору параболічного типу при КГВ буде також узагалънено-рекурентний простір параболічного типу. 
Доведення. Для того, щоб узагальнено-рекурентний простір параболічного типу $\left(V_{n}, g_{i j}, F_{i}^{h}\right)$ допускав нетривіальне КГВ на псевдорімановий простір $\left(\bar{V}_{n}, \bar{g}_{i j}\right)$, необхідно і достатньо виконання (3.1)-(3.4).

Залежність між коваріантними похідними афінора $F_{i}^{h}$ в просторах $V_{n}$ i $\bar{V}_{n}$ з урахуванням (3.1) та (3.3) має вигляд:

$$
F_{i \mid j}^{h}=F_{i, j}^{h}+\psi_{\bar{i}} \delta_{j}^{h}+\left(\phi_{\bar{i}}-\psi_{i}\right) F_{j}^{h},
$$

де "|" - знак коваріантної похідної в $\bar{V}_{n}$.

Після симетрування по індексах $i, j$ з огляду на (3.4) знайдемо:

$$
F_{(i \mid j)}^{h}=\tilde{q}_{(i} F_{j)}^{h}+\psi_{\bar{i}} \delta_{j}^{h}+\psi_{\bar{j}} \delta_{i}^{h}, \tilde{q}_{i}=q_{i}-\psi_{i}+\phi_{\bar{i}} .
$$

Опустимо в (3.6) індекс $h$ в просторі $\bar{V}_{n}$ і проциклюємо по $h, i, j . \mathrm{B}$ результаті отримаємо:

$$
\psi_{\bar{i}} \bar{g}_{h j}+\psi_{\bar{h}} \bar{g}_{j i}+\psi_{\bar{j}} \bar{g}_{i h}=0 .
$$

Згортання цієї рівності з $\bar{g}^{h j}$ по $h, j$ дає нам:

$$
\psi_{\bar{i}}=0
$$

і тому (3.6) набуває вигляду

$$
F_{(i \mid j)}^{h}=\tilde{q}_{(i} F_{j)}^{h},
$$

тобто афінор $F_{i}^{h}$ в просторі $\bar{V}_{n}$ також визначає узагальнено-рекурентну структуру параболічного типу.

Зауважимо, що із співвідношень (3.5) і (3.7) випливає

Наслідок 3.2. КГВ між узагалънено-рекурентними просторами параболічного типу $\left(V_{n}, g_{i j}, F_{i}^{h}\right),\left(\bar{V}_{n}, \bar{g}_{i j}, F_{i}^{h}\right)$ зберігае дивергенцію абіноpa, тобто

$$
F_{i, \alpha}^{\alpha}=F_{i \mid \alpha}^{\alpha} .
$$

$2^{\circ}$. Будемо казати, що КГВ узагальнено-рекурентного простору параболічного типу зберігає вектор узагальненої рекурентності, якщо при цьому

$$
\tilde{q}_{i}=q_{i},
$$

тобто співвідношення (3.8) набувають вигляду

$$
F_{(i \mid j)}^{h}=F_{(i, j)}^{h}=q_{(i} F_{j)}^{h} .
$$

3 (3.5) випливає, що за умови $\tilde{q}_{i}=q_{i}$ вектори $\psi_{i}$ і $\phi_{i}$ в основних рівняннях КГВ (3.1) пов'язані таким чином:

$$
\psi_{i}=\phi_{\bar{i}} .
$$


3 урахуванням цього зв'язку після згортання (3.1) по індексах $h, j$ маeмо:

$$
\bar{\Gamma}_{i \alpha}^{\alpha}=\Gamma_{i \alpha}^{\alpha}+(n+2) \psi_{i}
$$

Це свідчить про градієнтність вектора $\psi_{i}$, тобто існування функції

$$
\psi(x)=\frac{1}{2} \ln \left|\frac{\bar{g}}{g}\right|
$$

такої, що

$$
(n+2) \psi_{i}=\frac{\partial \psi(x)}{\partial x^{i}}
$$

$3^{\circ}$. Має місце

Теорема 3.3. Псевдорімановий K-nростір параболічного типу, який допускає КГВ зі збереженням інтегровної $K$-структури, необхідно $\epsilon$ келеровим.

Доведення. Узагальнено-рекурентна структура стає $K$-структурою, коли вектор узагальненої рекурентності дорівнює нулю, або породжує $K$-структуру $\tilde{F}_{i}^{h}=e^{-q} F_{i}^{h}$, коли $q_{i}$ є градієнтним. Отже розглянемо КГВ

$$
f:\left(V_{n}, g_{i j}, F_{i}^{h}\right) \rightarrow\left(\bar{V}_{n}, \bar{g}_{i j}, F_{i}^{h}\right)
$$

узагальнено-рекурентних просторів параболічного типу зі збереженням вектора узагальненой рекурентності $q_{i}$ за умов його градієнтності і інтегровності афінорной структури $F_{i}^{h}$. Тоді виконуються $(2.13),(2.14)$, (3.9), а тензори Рімана просторів $V_{n}$ i $\bar{V}_{n}$ з огляду на (2.5) та (2.6) задовольняють співвідношенням:

$$
\begin{aligned}
& R_{i j k}^{\bar{h}}-R_{\overline{i j k}}^{h}-R_{i \overline{j k}}^{h}-R_{i j \bar{k}}^{h}=0, \\
& \bar{R}_{i j k}^{\bar{h}}-\bar{R}_{\overline{i j k}}^{h}-\bar{R}_{i \bar{j} k}^{h}-\bar{R}_{i j \bar{k}}^{h}=0 .
\end{aligned}
$$

Знайдемо залежність між компонентами тензорів Рімана просторів $V_{n}$ i $\bar{V}_{n}$, застосовуючи $(3.1)$ i $(3.9)$ :

де позначено

$$
\begin{aligned}
\bar{R}_{i j k}^{h}= & R_{i j k}^{h}+\delta_{k}^{h} \psi_{i j}-\delta_{j}^{h} \psi_{i k}+F_{k}^{h} \phi_{i j}-F_{j}^{h} \phi_{i k}+ \\
& +F_{i}^{h} \phi_{[k j]}+\phi_{k} F_{i, j}^{h}-\phi_{j} F_{i, k}^{h}+\phi_{i} F_{[k, j]}^{h},
\end{aligned}
$$

$$
\psi_{i j}=\psi_{i, j}-\psi_{i} \psi_{j}, \quad \phi_{i j}=\phi_{i, j}-\phi_{i} \phi_{\bar{j}}-\phi_{j} \phi_{\bar{i}} .
$$

Із співвідношень (3.10) і (3.11) випливає рівність:

$$
\begin{aligned}
F_{i}^{h}\left(\phi_{\bar{j} k}-\right. & \left.\phi_{k \bar{j}}-\phi_{\bar{k} j}+\phi_{j \bar{k}}\right)+F_{j}^{h}\left(\phi_{\bar{i} k}+\phi_{i \bar{k}}\right)- \\
& \quad-F_{k}^{h}\left(\phi_{\bar{i} j}+\phi_{i \bar{j}}\right)-\psi_{k} F_{i, j}^{h}+\psi_{j} F_{i, k}^{h}-\psi_{i} F_{[k, j]}^{h}=0 .
\end{aligned}
$$


Опустимо тут індекс $h$ в $V_{n}$, просиметруємо по індексах $h, i$, а результат проциклюємо по $h, i, j$. Наразі маємо:

$$
F_{h k} b_{i j}+F_{i k} b_{j h}+F_{j k} b_{h i}=0
$$

де

$$
b_{i j}=\phi_{\overline{i j}}+\phi_{i \bar{j}}+\phi_{\overline{j i}}+\phi_{i \bar{j}}+\psi_{(i} q_{j)} .
$$

Після згортання (3.14) з $g^{k \alpha} A_{\alpha}^{h}$ по індексах $h, k$ (тензор $A_{i}^{h}$ ми ввели в попередньому розділі) виявляється, що $b_{i j}=0$, а звідси з огляду на $(2.3),(3.4),(3.9)$ та (3.12) випливає, що

$$
d_{i j}=-d_{j i}, \quad d_{i j}=\phi_{i \bar{j}}+\psi_{i j}
$$

Повернемось до (3.13), домножимо їх на $\phi_{h}$ і потім згорнемо по індексу $h .3$ урахуванням (3.4) та (3.15) отримаємо

$$
\psi_{j} d_{i k}+\psi_{k} d_{j i}+2 \psi_{i} d_{j k}=0 .
$$

Якщо $\psi_{i} \neq 0$ (КГВ не являється канонічним), існує вектор $a^{i}$ такий, що $a^{\alpha} \psi_{\alpha}=1$. Згортаючи (3.16) послідовно з $a^{i}$ по $i$ і з $a^{i} a^{j}$ по $i, j$ знаходимо $d_{i j}=0$, тобто з $(3.15)$ випливає

$$
\phi_{i \bar{j}}=-\psi_{i j}
$$

Використовуючи отримане співвідношення, перепишемо (3.13) у вигляді

$$
F_{k}^{h} \phi_{\alpha} F_{i, j}^{\alpha}-F_{j}^{h} \phi_{\alpha} F_{i, k}^{\alpha}-F_{i}^{h} \phi_{\alpha} F_{[j, k]}^{\alpha}-\psi_{k} F_{i, j}^{h}+\psi_{j} F_{i, k}^{h}-\psi_{i} F_{[k, j]}^{h}=0 .
$$

Після згортання з $A_{h}^{i}$ по $i$ і з $A_{h}^{j}$ по $j$ звідси отримуємо систему рівнянь, з якої певними алгебраїчними перетвореннями і з урахуванням (3.4) мaємо:

$$
\phi_{\alpha} F_{j, k}^{\alpha}=\psi_{j} q_{k}
$$

З огляду на цю рівність, (3.17) і те, що

$$
\phi_{\overline{i j}}=\psi_{i j}-\phi_{\alpha} F_{i, j}^{\alpha},
$$

запишемо (3.13) у вигляді:

$$
\psi_{i}\left(F_{[j, k]}^{h}-q_{k} F_{j}^{h}+q_{j} F_{k}^{h}\right)+\psi_{j}\left(F_{i, k}^{h}-q_{k} F_{i}^{h}\right)-\psi_{k}\left(F_{i, j}^{h}-q_{j} F_{i}^{h}\right)=0 .
$$

Опускаючи тут індекс $h$ в $V_{n}$ і симетруючи по $h, k$, знаходимо

$$
\psi_{k}\left(F_{h i, j}-q_{j} F_{h i}\right)+\psi_{h}\left(F_{k i, j}-q_{j} F_{k i}\right)=0 .
$$

Співставимо це рівняння з результатом його послідовного згортання 3 $a^{k}, a^{h}$ по $k, h$ відповідно і отримаємо:

$$
F_{i, j}^{h}=q_{j} F_{i}^{h} .
$$


Отже бачимо, що наша узагальнено-рекурентна структура $F_{i}^{h}$ параболічного типу виявилася рекурентно-параболічною [15]. Тоді з огляду на градієнтність $q_{i}$ в $V_{n}$ існує параболічно келерова структура

$$
\tilde{F}_{i}^{h}=e^{-q} F_{i}^{h} .
$$

\section{4. КГВ УЗАГАЛЬНЕНО-РЕКУРЕНТНИХ ПРОСТОРІВ ПАРАБОЛІЧНОГО ТИПУ ЗІ ЗБЕРЕЖЕННЯМ ВЕКТОРА УЗАГАЛЬНЕНОЇ РЕКУРЕНТНОСТІ НА ПЛОСКИЙ ПРОСТІР}

$1^{\circ}$. Нехай узагальнено-рекурентний простір параболічного типу з інтегровною афінорною структурою $\left(V_{n}, g_{i j}, F_{i}^{h}\right)$ допускає квазі-геодезичне відображення зі збереженням вектора узагальненої рекурентності на плоский простір $\bar{V}_{n}=E_{n}$. Отже $\bar{R}_{i j k}^{h}=0 \mathrm{i}$

$$
\bar{R}_{i j k}^{\bar{h}}-\bar{R}_{\overline{i j k}}^{h}-\bar{R}_{i \bar{j} k}^{h}-\bar{R}_{i j \bar{k}}^{h}=0 .
$$

Тоді у відповідності з Теоремою 2.4 вектор узагальненої рекурентності $q_{i}$ простору $\bar{V}_{n}-$ градієнтний.

Оскільки за умовою наше КГВ зберігає вектор узагальненої рекурентності, а афінорна структура у нас вважається інтегровною, то за Теоремою $3.3\left(V_{n}, g_{i j}, F_{i}^{h}\right)$ є рекурентно-параболічним простором.

Враховуючи ці обставини і спираючись на [15, Теорема 2], приходимо до висновку, що має місце

Теорема 4.1. Якщо узагалънено-рекурентний простір параболічного mипу $\left(V_{n}, g_{i j}, F_{i}^{h}\right)$ з інтегровною афінорною структурою $F_{i}^{h}$ допускае нетривіальне КГВ зі збереженням вектора узагалъненої рекурентносmi $q_{i}$ на плоский простір $\bar{V}_{n}=E_{n}$, то $V_{n}$ буде Річчі-плоским, симетричним, тобто

$$
\begin{gathered}
R_{i j}=0, \\
R_{i j k, l}^{h}=0,
\end{gathered}
$$

вектор $q_{i}$ - градіентним,

$$
q_{i}=\frac{\partial q(x)}{\partial x^{i}}
$$

а тензор Рімана простору $V_{n}$ необхідно має вигляд

$$
R_{h i j k}=C e^{-2 q(x)}\left(F_{h k} F_{i j}-F_{h j} F_{i k}+2 F_{h i} F_{k j}\right)
$$

при деякій сталій $C$. 
$2^{\circ}$. В [15] для рекурентно-параболічного простору, тензор Рімана якого має структуру (4.1), отримано компоненти метричного тензора в околі деякої точки $M_{\circ}$ в $V_{n}$ :

$$
g_{i j}(y)=\stackrel{\circ}{g}_{i j}+\frac{C e^{-2 q^{\circ}}}{8} y^{\alpha} \stackrel{\circ}{F}_{\alpha i} y^{\beta} \stackrel{\circ}{F}_{\beta j},
$$

де $\stackrel{\circ}{g}_{i j}, \stackrel{\circ}{F}_{i j}, \stackrel{\circ}{q}$ - компоненти тензорів $g_{i j}, F_{i j}$ і функції $q$ в точці $x_{\circ}, y^{h}$ ріманові координати з початком в точці $x_{\circ}$.

Отже спираючись на Теореми 3.3 і 4.1, робимо висновок, що справедлива

Теорема 4.2. Якщо узагалънено-рекурентний простір параболічного muny $\left(V_{n}, g_{i j}, F_{i}^{h}\right)$ з інтегровною афінорною структурою $F_{i}^{h}$ допускає нетривіалье КГВ зі збереженням вектора узагальненої рекурентносmi $q_{i}$ на плоский простір $\bar{V}_{n}=E_{n}$, то його метричний тензор в околі деякої точки $M_{\circ}$ в $V_{n}$ може бути представлений у вигляді (4.2).

\section{5. Висновок}

В статті введено поняття узагальнено-рекурентної афінорної структури на псевдорімановому просторі. До таких структур відносяться, наприклад келерові і $K$-структури. Почато дослідження квазі-геодезичних відображень псевдоріманових просторів параболічного типу. Розглянуто тільки такі відображення, які зберігають вектор узагальненої рекурентності, хоча найбільш інтересні і різноманітні результати слід очікувати якраз в протилежному випадку. Виявилося, що якщо псевдорімановий простір з інтегровною $K$-структурою допускає нетривіальне КГВ, то цей простір - параболічно-келеровий. В узагальнено-рекурентному просторі параболічного типу, який допускає нетривіальне КГВ на плоский простір, також існує параболічно-келерова структура, тому висновки останнього розділу статті цілком узгоджуються з результатами, які представлені в [9].

\section{ЛiтератУРА}

[1] V. E. Berezovskii, J. Mikeš. Almost geodesic mappings of spaces with affine connection. J. Math. Sci. (N.Y.), 207(3):389-409, 2015, doi: 10.1007/s10958-015-2378-5. Translated from Itogi Nauki Tekh. Ser. Sovrem. Mat. Prilozh. Temat. Obz. Vol. 126, Geometry, 2013.

[2] V. E. Berezovskiı̌, I. Mikesh, G. Khuda, E. E. Chepurnaya. Canonical almost geodesic mappings that preserve the projective curvature tensor. Russian Mathematics, 61(6):15, 2017, doi: 10.3103/s1066369x17060019.

[3] Ryszard Deszcz, Mileva Prvanović. Holomorphically projective mappings onto semisymmetric anti-Kähler manifolds. Tensor (N.S.), 75(1):9-28, 2014. 
[4] V. Kiosak, A. Savchenko, T. Shevchenko. Holomorphically projective mappings of special kähler manifolds. AIP Conference Proceedings, 2018, doi: 10.1063/1.5064924.

[5] Josef Mikeš, Alena Vanžurová, Irena Hinterleitner. Geodesic mappings and some generalizations. Palacký University Olomouc, Faculty of Science, Olomouc, 2009.

[6] J. Mikeš, E. Stepanova, A. Vanžurová, S. Bácsó, V.E. Berezovski, O. Chepurna, M. Chodorová, H. Chudá, M.L. Gavrilchenko, M. Haddad. Differential geometry of special mappings. Palacký University Olomouc, Faculty of Science, Olomouc, 2015.

[7] Miloš Z. Petrović, Mića S. Stanković. Special almost geodesic mappings of the first type of non-symmetric affine connection spaces. Bull. Malays. Math. Sci. Soc., 40(3):13531362, 2017, doi: 10.1007/s40840-015-0118-0.

[8] P. Peška, J. Mikeš, H. Chudá, M. Shiha. On holomorphically projective mappings of parabolic Kähler manifolds. Miskolc Math. Notes, 17(2):1011-1019, 2016, doi: 10.18514/MMN.2017.1893.

[9] Mohsen Shiha, Josef Mikeš. On holomorphically projective flat parabolically-Kählerian spaces. 250:467-474, 2006.

[10] Mića S. Stanković, Milan L. Zlatanović, Nenad O. Vesić. Basic equations of Galmost geodesic mappings of the second type, which have the property of reciprocity. Czechoslovak Math. J., 65(140)(3):787-799, 2015, doi: 10.1007/s10587-015-0208-z.

[11] Д. В. Беклемишев. Дифберенииальная геометрия пространств с почти комплексной структурой. Итоги науки: Геометрия, 1963. Москва: ВИНИТИ, 1965.

[12] И. Н. Курбатова. Квази-геодезические отображения римановых пространств. $\mathrm{PhD}$ thesis, 1980.

[13] И. Н. Курбатова. Канонические квази-геодезические отображения параболически кэлеровых пространств. Proc. Intern. Geom. Center, 7(1):53-64, 2014.

[14] И. Н. Курбатова. О закономерностях канонических квази-геодзических отображений параболически кэлеровых пространств. Proc. Intern. Geom. Center, 7(2):26-35, 2014.

[15] И. Н. Курбатова, О. Т. Сисюк. Квази-геодезические отображения рекуррентнопараболических пространств. Proc. Intern. Geom. Center, 8(1):74-83, 2014.

[16] А. З. Петров. Моделирование физических полей. Гравитация и теория относительности, (4-5):7-21, 1968.

[17] Н. С. Синюков. Геодезические отображения римановых пространств. М.: Наука, 1979.

[18] Н. С. Синюков. Почти геодезические отображсения афбинносвязных и римановых пространств, volume 13 of Итоги науки и техники: Проблемы геометрии. Москва: ВИНИТИ, 1982.

Надійшла до редакщї 1 серпня 2020, прийнята до друку 29 вересня 2020.

Курбатова I.M.

ОНУ, ОДЕСА, УКРАЇнА

Email: irina.kurbatova27@gmail.com

Піструіл М.I.

ОНУ, ОДЕСА, УКРАЇнА

Email: margaret.pistruil@gmail.com 